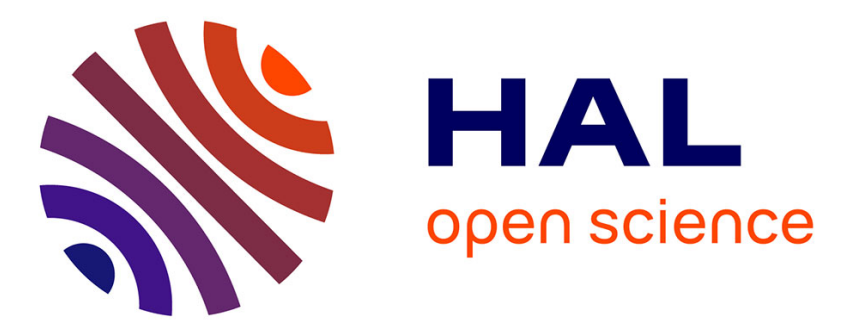

\title{
Un graffite grec (évaluation de contenance) sur une cruche gallo-romaine à Châtillon-sur-Seiche (Ille-et-Vilaine)
}

Michel Lejeune

\section{- To cite this version:}

Michel Lejeune. Un graffite grec (évaluation de contenance) sur une cruche gallo-romaine à Châtillonsur-Seiche (Ille-et-Vilaine). Gallia - Fouilles et monuments archéologiques en France métropolitaine, 1993, 50, pp.223-225. 10.3406/galia.1993.2939 . hal-01913125

\section{HAL Id: hal-01913125 \\ https://hal.science/hal-01913125}

Submitted on 29 Jan 2020

HAL is a multi-disciplinary open access archive for the deposit and dissemination of scientific research documents, whether they are published or not. The documents may come from teaching and research institutions in France or abroad, or from public or private research centers.
L'archive ouverte pluridisciplinaire HAL, est destinée au dépôt et à la diffusion de documents scientifiques de niveau recherche, publiés ou non, émanant des établissements d'enseignement et de recherche français ou étrangers, des laboratoires publics ou privés.

\section{(ㅇ)(1) $\$$}

Distributed under a Creative Commons Attribution - NonCommercial - NoDerivatives| 4.0 


\title{
Un graffite grec (évaluation de contenance) sur une cruche gallo-romaine à Châtillon-sur-Seiche (Ille-et-Vilaine)
}

\author{
par Michel LEJEUNE *
}

Sur une cruche de fabrication locale, dans une villa gallo-romaine voisine de Rennes, au II $^{\mathrm{e}}$ s. après J.-C. a été inscrite à la pointe après cuisson une indication de contenance en langue et écriture grecques.

In a gallo-roman villa near Rennes a local hand at some time in the 2nd c. A.D. inscribed a locally-made jar with a greek graffilo stating its capacity.

Mots clés : Armorique gallo-romaine, épigraphie grecque, litra (volume d'huile), métrologie grecque, Riedones.

Au lieu-dit La Guyomerais, sur la commune de Châtillon-sur-Seiche, à environ $6 \mathrm{~km}$ au sud de Rennes, une vaste villa gallo-romaine, fouillée d'avril 1984 à octobre 1987 par l'équipe d'Alain Provost $^{1}$, a livré un important matériel ${ }^{2}$. Parmi les céramiques de fabrication locale figure une cruche ins-

* Membre de l'Institut, 25, rue Gazan, 75014 Paris.

1 Fouilles signalées : après la première campagne dans les Informations archéologiques, Circonscription de Bretagne, Gallia, 43, 1985, 2, p. 290-292; après la clôture du chantier, dans Gallia Informations, 1990, 1-2, p. 42-46. De plus, voir la notice parue dans G. Leroux, A. Provost, Carte archéologique de la Gaule, L'Ille-et-Vilaine 35 (= CAG 35. 347), Paris, 1990, p. 270-274.

2 Une partie en a été présentée à l'écomusée du Pays de Rennes dans l'exposition (juin-décembre 1990) «Nos ancêtres les Riedons". Le catalogue (dû à A. Provost) ne fait (p. 35) qu'une brève allusion (sans illustration) à l'objet ici commenté. crite $^{3}$, brisée et lacunaire, mais de profil reconstituable ${ }^{4}$ : vase à pâte bistre, très galbé, avec double cannelure en haut de panse. L'objet, trouvé dans un dépotoir, est attribué par A. Provost au II ${ }^{\mathrm{e}}$ s. après J.-C.

Il porte sous le col, gravé à la pointe après cuisson, un graffite en langue et écriture grecques ${ }^{5}$; caractères de 8 à $12 \mathrm{~mm}$ dont le haut s'accroche à la

3 Je dois mon information, et aussi bien les dessins ici reproduits, à Alain Provost, que j'ai plaisir à remercier.

4 La hauteur subsistante (pied et col brisés) excède $29 \mathrm{~cm}$; le diamètre de la panse mesure $27 \mathrm{~cm}$; les diamètres du pied et de l'embouchure (perdus) devaient être d'une dizaine de centimètres.

5 Par ailleurs, à La Guyomerais, rares et courts graffites sur terre cuite en langue et écriture latines (p. 34-35 du catalogue d'exposition) : brève liste de noms d'hommes; abréviation ou exclamation $A V E$; etc. - Latin aussi est le graffite sur plaque décorative en schiste, illustré p. 34 . 


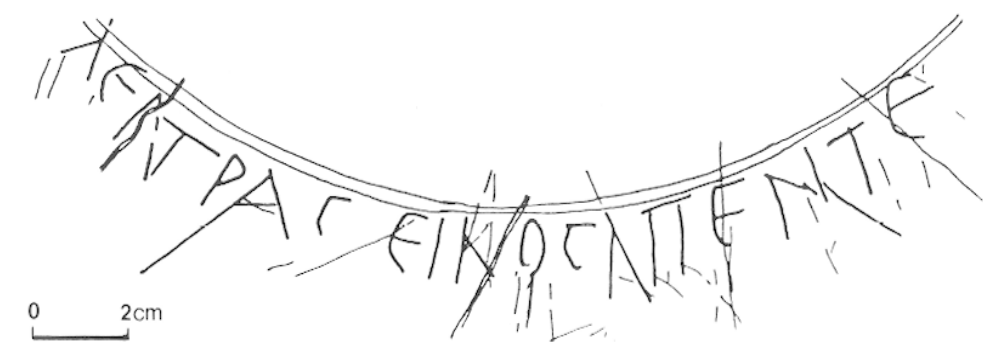

Fig. 1 -

Le graffite de Châtillon-sur-Seiche. cannelure inférieure; écriture hellénistique $(\varepsilon, \sigma$ lunaires), régulière et soignée; texte complet à gauche et à droite, avec blanc assez net après le premier mot (fig. 1). Lecture :

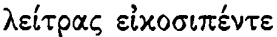

ce qui signifie ${ }^{6}$ : "(ce récipient contient) vingt-cinq litras " $"$.

Il n'existe pas d'épigraphie grecque d'Armorique. Isolé dans cette région, et isolé sur le site même, un tel document ne peut que surprendre.

Céramique de fabrication locale : le graffite a donc été gravé sur place.

Il est douteux qu'il soit dû à un potier façonnant sur commande des récipients de contenance déterminée; outre que l'indication volumétrique eût alors probablement été gravée avant cuisson, un coefficient 25 serait tout à fait insolite en métrologie.

Il faut plutôt y voir une intervention de l'utilisateur, en l'espèce l'intendant (actor) du domaine. Contenance constatée a posteriori, et notée pour qu'on n'eût pas à la remesurer. L'huile en jarres que reçoivent les magasins de la villa, il faut, pour la redistribuer, des récipients plus maniables, de moindres dimensions, et il est commode que leurs capacités soient, une fois pour toutes, jaugées et inscrites. D'une telle procédure d'étalonnage domestique apparaît donc ici un témoin.

6 Consulté par le fouilleur, Léon Fleuriot n'avait pas identifié le premier mot et n'avait donc pas vu qu'il s'agit d'une indication de contenance; il se bornait à traduire : "la vingt-cinquième" [sic]. A propos du graffite, A. Provost se contente de mentionner l'avis de L. Fleuriot (Catalogue, p. 35 ; CAG 35. 347, p. 271).

7 Avec une orthographe tardive (-8เ- pour -t-), nom d'unité (de poids; puis de volume d'huile). Plutôt que de le traduire, je préfère le transposer en "litra». Sur l'histoire de ce terme, voir Revue des Etudes Grecques, 106, 1993, p. 1-11.
Mais pourquoi une inscription en grec? - $\mathrm{Ou}$ bien fantaisie ludique d'un Gallo-romain un peu hellénomane. - Ou bien l'intendant se trouvait être un Graeculus que les hasards de l'existence (sait-on jamais ?) auraient mené jusqu'à cette ferme du pays redon et qui, tout romanisé qu'il fût certainement en tel environnement, n'en usait pas moins, à l'occasion, de son grec quand il voulait noter quelque chose à son usage. - De toute façon, l'explication se situe au niveau d'un individu (pour nous anonyme) et nous échappe.

Par ailleurs (de façon, il est vrai, ambiguë, on va le voir) le graffite de Châtillon pourrait mettre en cause la métrologie.

On enseigne que la $\lambda i$ iтpa, volume liquide (spéci-

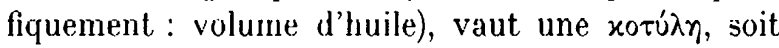
environ 27 de nos centilitres. I a contenance ici chiffrée serait donc de quelque $675 \mathrm{cl}$. Est-ce compatible avec notre poterie?

L'objet est malheureusement incomplet (embouchure et pied, notamment, nous manquent); mais de l'essentiel du corps, le profil est restituable, comme le montre le dessin d'A. Provost; et du profil on sait déduire le volume ${ }^{8}$. J'ai là recouru, par deux fois, à la compétence de Guy Bertucchi ${ }^{9}$.

Dans un premier temps, entre un niveau inférieur $\mathrm{A}$, de détermination à peu près assurée, et le niveau $B$ qu'atteindrait le liquide si le remplissage était total (fig. 2), G. Bertucchi a évalué le volume de liquide à $895 \mathrm{cl}$ (ce qui porterait la litra à $35,8 \mathrm{cl}$, valeur supérieure d'un tiers à ce qui est communément admis). Je lui ai alors demandé où devraient se situer des niveaux $A^{\prime}$ et $B^{\prime}$ pour un volume de

8 D'un vase fait au tour (dont toutes les sections horizontales sont donc circulaires), la géométrie permet de calculer le volume à partir de la coupe verticale.

9 Conservateur détaché au Centre Camille Jullian $(\Lambda \mathrm{ix}$ en-Provence), que je remercie vivement de son concours. 


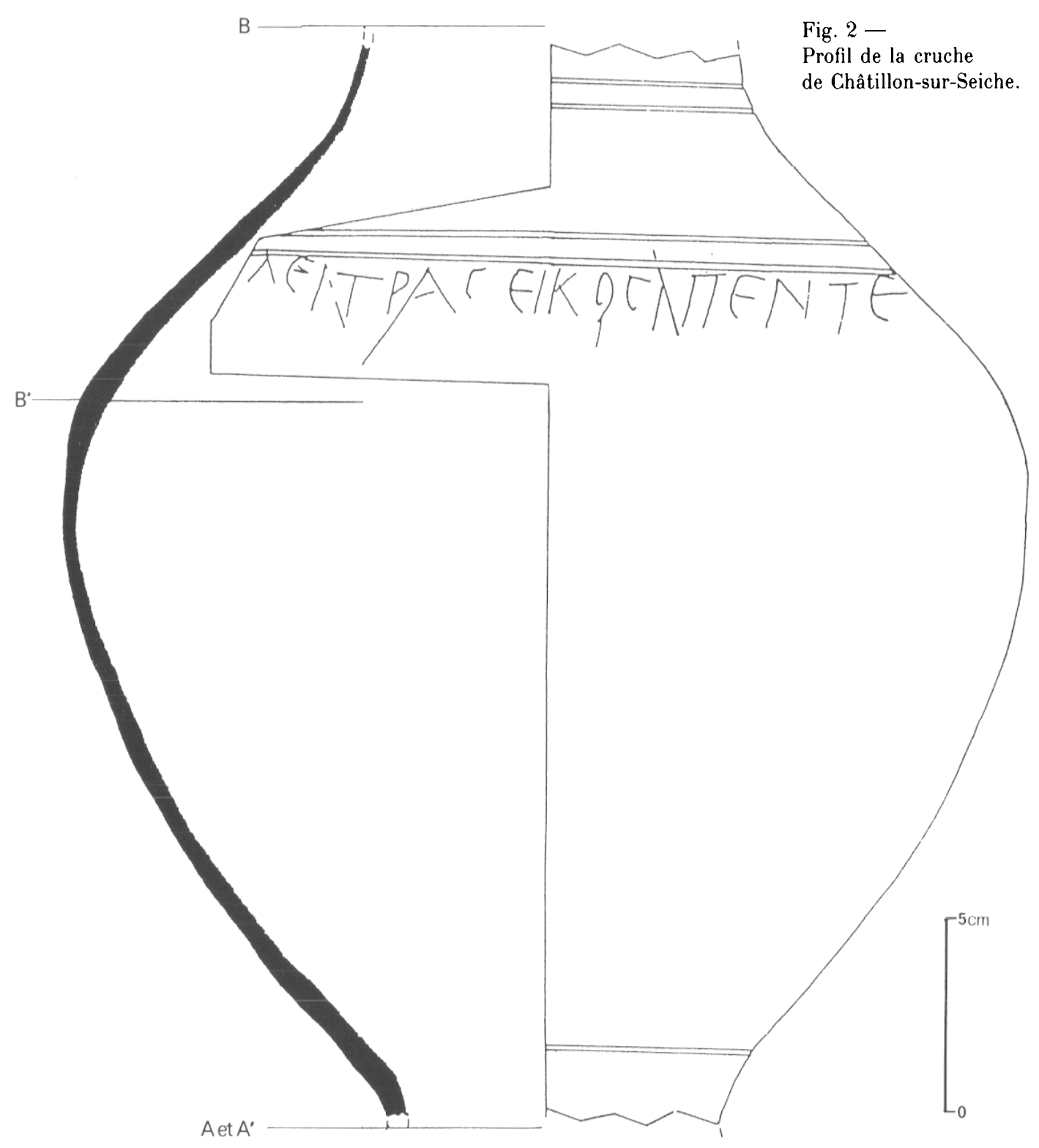

$675 \mathrm{cl}$; le bas $\left(\mathrm{A}^{\prime}=\mathrm{A}\right)$ n'étant guère, pour lui, en discussion, il a déterminé $\mathrm{B}^{\prime}$ comme sis en haut de panse, à quelque $4 \mathrm{~cm}$ au-dessous de la cannelure inférieure. Adhuc sub judice..., en attendant peutêtre un recours à l'objet lui-même, ou du moins à une réplique de l'objet, avec restitution matérielle plausible des portions manquantes, si ce n'est pas une chimère.

A moins, enfin, qu'il s'agisse d'une notation occasionnelle (enregistrement de transvasement, un beau jour, d'unc certaine quantité d'huile, pré-mesurée) et non d'une inscription à vocation permanente (caractéristique volumétrique du pot), ce qui ferait disparaître tout problème métrologique. Mais notre intendant eût-il, en ce cas, tracé une élégante inscription en toutes lettres au lieu d'un bref sigle chiffré?

Michel Lejeune

\section{Origine des documents}

Les illustrations sont dues à A. Provost; les repères A, $A^{\prime}, B$ et $B^{\prime}$ de la figure 2 ont été ajoutés par $G$. Bertucchi. 